\title{
Zur Differenzierung des Wortschatzes im Bereich der Berufsbezeichnungen im heutigen Deutsch
}

\author{
Olga Geislerová
}

This article presents the results of an exploratory empirical study into the formation of new names of occupations and jobs in German language. In connection with the development of the human society and new technologies there are new occupations and their names. Lexical units have their own conditions on derivation and compounding. A variety of examples from contemporary language production (journals, newspapers, websites) are presented.

The formation of new names of occupations and jobs - conditions on derivation and compounding - knowledge of morphology - semantic side of a lexical unit - connotations knowing the semantic value of the word - precision of meaning

Neubildungen für Berufsbezeichnungen - Derivationsbedingungen - Kompositabildung morphologisches Wissen - Semantik von Lexemen - Bedeutungspräzisierung

Mit der Gesellschaft verändert sich auch die Sprache: Von einer plakativen, emotional geprägten Sprache hin zu einer nüchternen, fachlichen, juristisch und pragmatisch geprägten Sprache. Wörterbücher sind keine Handbücher der Geistesund Sozialgeschichte. Dennoch geben die zahlreichen Personenbezeichnungen einen Einblick in die Figurenwelt der vergangenen und gegenwärtigen Gesellschaft. Der Arbeitsmarkt - eine Bildung, die semantisch Angebot und Nachfrage signalisiert wird heute überwiegend durch die Prägungen Arbeitgeber-Arbeitnehmer geteilt, die sprachlich eine ganz andere Perspektive darlegen als das Paar Unternehmer-Arbeiter. Durch die beiden Komposita mit Arbeit als erstem Glied wird sprachlich eine Verbindung geschaffen, wodurch die beiden Parteien als gleichgewichtige Größen gegenübergestellt werden. Zum Wortpaar Arbeitgeber und Arbeitnehmer gehört eine ganze Reihe anderer Personenbezeichnungen, die aber zumeist auf eine der beiden Positionen bezogen sind und bleiben. Vor allem die Position der Arbeitgeber kennt viele andere Bezeichnungen, die von „normalsprachlich“ bis „abwertend“ reichen: Fabrikbesitzer, Fabrikherr, Fabrikant, Großindustrieller, Brotherr (veraltet), Brötchengeber (scherzhaft); Profitmacher, Profitjäger, Kapitalist (abwertend). Auf der Arbeitnehmerseite gibt es den Lohnempfänger, den Lohnabhängigen, sogar den Lohnsklaven (abw.) Hinzu kommen viele Berufsbezeichnungen mit dem Grundwort Arbeiter: Fabrikarbeiter, Lohnarbeiter, Akkordarbeiter usw.

Zweiseitige Gruppenbezeichnungen sind auch auf anderen Ebenen zu finden und können leicht zu kontrastiven Einschätzungen führen. Die wissenschaftliche Soziologie verwendet die Einteilung in "Schichten“. Die Soziolinguistik hat deshalb neue Personenbezeichnungen entwickelt: Mittelschicht, Unterschicht, Mittelschichtkind, Unterschichtkind. 
Im beruflichen Bereich findet man im Zusammenhang mit der Veränderung der Arbeitswelt und mit der Entwicklung der EDV-Technologien immer wieder neue Berufsbezeichnungen, die ab und zu nur dem „eingeweihten“" also spezialisierten Arbeitsmilieu bekannt sind. Wo man sich früher mit einigen wenigen Bezeichnungen begnügen konnte, erscheinen heute viele verschiedene und vielversprechende Benennungen. Als Beispiel kann man auf die Berufsbezeichnungen im Anzeigenbereich „Verkauf und Vertrieb“ hinweisen. Wenn man die Stellenangebote in den Zeitungen liest, findet man Dutzende Benennungen für Männer und Frauen, die beruflich den Verkauf von verschiedenen Produkten vorbereiten, planen und praktizieren. Da ist viel Wirtschaft, Marketingforschung und Psychologie im Spiel. Deshalb genügt nicht mehr, nur von Verkäufern und Verkäuferinnen zu sprechen, vielmehr sind andere Bezeichnungen gefragt: Verkaufsberater, Verkaufsprofi, Verkaufssachberater, Verkaufsbeauftragter, Verkaufsrepräsentant, Verkaufsspezialist, Verkaufsleiter, Fachverkäufer, Technischer Verkäufer, Direktverkäufer, Profiverkäufer, Gebrauchswagenverkäufer, Immobilienverkäufer. Neben dem Grundwort Verkauf scheint auch die Wortbildung mit Vertrieb recht produktiv zu sein: Vertriebsmitarbeiter, Vertriebsbeauftragter, Vertriebsprofi, Vertriebsleiter, Vertriebsmann, Vertriebsassistent, Vertriebsingenieur. Auch im Aufgabenbereich der Verkaufsleitung kommen viele Personenbezeichnungen vor: Leiter, Filialleiter, Vertriebsleiter, Gebietsleiter, Verkaufsleiter, Organisationsleiter, Abteilungsleiter, Teamleiter, Projektleiter, Gesamtbetriebsleiter. Selten findet man die Benennung Handelsvertreter.

Außer den erwähnten Beispielen kommen noch andere Bildungstypen vor:

- berater: Fachberater, Verkaufsberater, Immobilienberater, Urlaubsberater

- profi: Verkaufsprofi, Vertriebsprofi, Außendienstprofi, Profiverkäufer

- beauftragter: Vertriebsbeauftragter, Verkaufsbeauftragter, Datenschutzbeauftragter

- manager: Projektmanager, Immobilienmanager, Seminar-Manager

-kraft: Fachkraft, Lehrkräfte, Vollzeitkräfte, Regalservicekräfte, Reinigungskräfte, Touristik-Fachkraft

-mann / -fachmann: Vertriebsmann, Marketingfachmann, Versicherungskaufmann

Diese Grundwörter dienen zur Reihenbildung von neuen Wörtern und deshalb könnte man sie bereits als Halbsuffixe bezeichnen.

Die Assistenten und Assistentinnen arbeiten nicht nur an der Universität, sondern sie werden auch für die Abteilung Verkauf/Vertrieb gesucht: Vertriebsassistent, Büroassistent, Werbeassistent. Zu den auffälligen Besonderheiten dieser Branche gehören Bildungen wie Absatzförderer, Teilzeitstimme (für die Telefonwerbung) oder Propagandist (für Messetätigkeit).

Auf moderne Berufe sind die folgenden Suchanzeigen bezogen: Programmierer, Informatiker, Technikinformatiker, Betriebsinformatiker, EDV-Fachmann, EDV-Profi, EDV-Gruppenleiter, Software-Entwickler, Hardware-Entwickler, EDV-Kaufmann, Systemanalytiker. 
Was die Führungspositionen/Führungskräfte betrifft, kommen Zusammensetzungen mit dem Grundwort Leiter, Manager und Geschäftsführer vor:

Leiter: Bauleiter, Oberbauleiter, Technischer Leiter, Bezirksleiter, Regionalleiter, Exportleiter, Niederlassungsleiter, Betriebsleiter, Werkleiter, Produktionsleiter, Personalleiter, Konstruktionsleiter, Baumarktleiter usw.

Manager: Office Manager, Product Manager, Sales-Manager, Account Manager, Key-Account-Manager, Marketing-Manager, Business-Manager, Gebäudemanager, Forschungsmanager, Datenmanager usw.

Anhand der neuen Berufsbezeichnungen sind vor allem die Phänomene der Differenzierung und Umwertung zu beobachten. Der Prozess der sachlichen Differenzierung der Berufswelt sowie der sprachlichen Motivierung und Umwertung ist weiter fortgeschritten. Umwertung heißt, jedenfalls auf der Ebene der Stellenanzeigen, soviel wie „Aufwertung der Benennungen“. Das Wort Techniker allein besagt, dass jemand Fachmann im Bereich der Technik ist. Der Ausbildungsaspekt tritt in den Hintergrund. In den vielen Benennungen (Hochbautechniker, Nachrichtentechniker) findet nicht nur eine Differenzierung und Motivierung statt, sondern in vielen Beispielen auch eine soziale und sprachliche Aufwertung (Papiertechniker, Holztechniker, Farbtechniker), obwohl bei solchen Beispielen nicht immer zu erkennen ist, gegenüber welchen Wörtern die Aufwertung stattgefunden hat. Man darf nicht übersehen, dass hinter den ansprechenden Benennungen auch anspruchsvolle Erwartungen der Inserenten stehen. Daher sollen die sozial aufgewerteten Tätigkeiten die Bewerber auch psychologisch motivieren.

Die meisten neuen Berufsbezeichnungen sind als Wortbildungskonstruktionen entstanden, also vorwiegend handelt es sich um Zusammensetzungen (Komposita) und Ableitungen (Derivativa). Sprachtypologisch kann man feststellen, dass das Deutsche sich im Bereich des Wortschatzes zunehmend zu einer Wortbildungssprache entwickelt. Die personalen Komposita lassen sich zu etwa 90 Prozent als Determinativkomposita charakterisieren. Sie heißen auch Bestimmungskomposita, weil das Grundwort von einem Bestimmungswort näher modifiziert wird: Ein Krankenhausarzt ist ein Arzt, der in einem Krankenhaus tätig ist. Formal und semantisch ähnlich sind die folgenden Beispiele: Schularzt, Hausarzt, Gefängnisarzt, Werkarzt, Schiffsarzt usw. Daneben gibt es Komposita mit anderen semantischen Verhältnissen: Das Bestimmungswort erklärt den Fachbereich desjenigen Arztes, wie z. B. Kinderarzt, Frauenarzt, Tierarzt, Augenarzt, Zahnarzt, Hautarzt, oder seine Stellung und Funktion: Chefarzt / Oberarzt, Assistenzarzt / Sekundararzt.

Strukturell bieten die Determinativa folgende Möglichkeiten:

Nomen/Nomen: Deutschlehrer, Klassenlehrer, Klavierlehrer, Sportlehrer, Tanzlehrer, Hochschullehrer, Personalberater, Steuerberater, Systemberater, Vermögensberater, Versicherungsberater, Unternehmensberater Adjektiv/Nomen: Junglehrer, Privatlehrer Verb/Nomen: Reitlehrer, Turnlehrer, Rennfahrer 
Partikel/Nomen: Außenhändler, Innenarchitekt, Nurnotar, Nochpräsident (Putin), Mitarbeiter

Die Konstruktionen der Determinativa stehen in einem Verhältnis der Subordination, der Unterordnung.

Die Kopulativa (Kopulativkomposita) sind morphologisch und semantisch anders zu verstehen. Beide Bestandteile sind gleichwertig, die Beziehung zwischen ihnen ist als Koordination (Nebenordnung) anzusehen. Das Vorkommen von Kopulativa ist im Vergleich zu den oben genannten Determinativa auf eine geringe Anzahl beschränkt. In der Fachliteratur werden immer wieder die gleichen Beispiele genannt: Arztkosmonaut, Dichterkomponist, Fürstbischof (BRAUN: 1997).

Im heutigen Deutsch findet man eine ganze Reihe von Ableitungen, die viele neue Berufsbezeichnungen mit Hilfe von Suffixen ausdrücken. An den Ableitungsprozessen sind vor allem folgende Suffixe beteiligt:

-er - verbale Basis: Das Suffix -er bildet mit Verben Nomina agentis (von lat. agens „Handelnder"): Der Prüfer ist jemand, der prüft. Weitere Spezifizierung ermöglicht die Zusammensetzung mit weiteren Bestimmungswörtern: Buchprüfer, Technikprüfer, Wirtschaftsprüfer. Im Prinzip sind alle Verben, die in ihrem Aktantenrahmen ein Agens enthalten, mit -er ableitbar: Arbeiter, Dichter, Denker, Lehrer, Maler, Pfleger, Spieler. Interessant sind die Wörter Empfänger, Sender, Drucker, Ordner, die nicht nur Personen, sondern auch Gegenstände bezeichnen, die sog. Nomina instrumenti. Verben, die dagegen keinen Erstaktanten (prototypisch: ein Agens), sondern nur einen Zweitaktanten (Patiens bzw. Vorgangsträger) implizieren, können keine Nomina agentis bilden: *Ankommer, ${ }^{*}$ Faller, ${ }^{*}$ Wachser, ${ }^{*}$ Ersticker, ${ }^{*}$ Einschläfer (GREWENDORF: 1989, 21 f.; zu analogen Verhältnissen im Tschechischen ${ }^{*}$ zkolabovač - vgl. KARLÍK: 2004, 74).

-er - substantivische Basis: Der Handwerker ist jemand, der ein Handwerk ausübt. Diese substantivischen Ableitungen sind jedoch bei Weitem nicht so oft vertreten wie es bei der oben genannten Gruppe der Fall ist, diese Wortbildungsart gilt als „invital“ (unproduktiv). Weitere Beispiele sind der Tabelle unten zu entnehmen.

\begin{tabular}{|l|l|}
\hline \multicolumn{2}{|l|}{ Ableitungen mit -er } \\
\hline von verbalen Basen & von substantivischen Basen \\
\hline Anlagebauer, Modellbauer & Gärtner \\
Betreuer - DV-Betreuer & Geriatriker \\
Designer - Modedesigner, Screendesigner & Glaser \\
Erzieher & Grafiker \\
Entsorger & Grenzer (gespr.): Grenzbeamter \\
Fliesenleger & Händler - Außenhändler, Blumenhändler, \\
Formelfahrer & Buchhändler, Verlagsbuchhändler, \\
Forscher - Genforscher, Marktforscher & Mobilfunkhändler, Südfrüchtehändler \\
Leiter - Reiseleiter & Hotliner \\
Mediendidaktiker & Informationsbroker, Informationstechniker \\
\hline
\end{tabular}




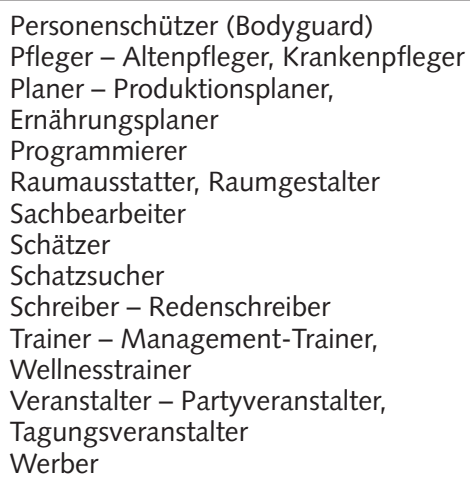

Logistiker

Mediendidaktiker

Mechatroniker

Netzwerker

Öko-Baumeister

Repromediziner

Techniker - DV-Techniker, Elektrotechniker, Haustechniker, Mikrotechniker,

Servicetechniker, Solartechniker

Treasurer (Schatzmeister)

Web-Texter

Wissenschaftler/Wissenschafter

Daneben werden auch noch weitere, nicht besonders produktive Derivationssuffixe verwendet:

-ler - substantivische Basis: Sport-Sportler

Makler, Immobilienmakler

Tischler

Taxler (gespr. Taxifahrer)

Mittelständler (Angehöriger des Mittelstandes)

Ruheständler (jd., der im Ruhestand ist)

-ner - substantivische Basis: Pförtner, Rentner

-iker - substantivische Basis: Akademiker, Musiker, Philharmoniker

-ist - substantivische Basis: Allround-Journalist, Cellist, Florist, Haarstylist, Halbfinalist, Fußball-Bundesligist (ugs.), Polizist, Visagist, WEB-Konzeptionist

-ist - verbale Basis: Komponist, Publizist, Spezialist, CAD-Spezialist, Marketingspezialist

-ent - verbale Basis: Dirigent, Disponent, Korrespondent, Referent, Pharmareferent

-eur: Kontrolleur/Controller, Friseur, Netzprojekteur, Masseur, Monteur, Trockenbaumonteur, Redakteur, Online-Redakteur, Technischer Redakteur, Ingenieur, Finanzingenieur, Gartenbauingenieur, Vertriebsingenieur

-eut: Physiotherapeut, Psychotherapeut

-ier: Bankier, Hotelier

-oge: Allergologe, Evolutionsbiologe, Pädagoge, Psychologe

-or: Direktor, Drehbuchautor, Inspektor, Mediator, Teletutor

Einige Personenbezeichnungen kann man als Zusammenbildungen analysieren. Auf den ersten Blick sehen Bildungen wie Gesetzesbrecher oder Rekordhalter wie Zusammensetzungen aus. Ihre Aufösung zeigt aber folgendes: Der Rekordhalter ist nicht „Halter eines Rekords“, sondern „jemand, der einen Rekord hält“. Beispiele dieses Bildungstyps werden in der Wortbildungslehre den Ableitungen zugerechnet. 
Der Bildungstyp ist aber so produktiv, dass die Beispielgruppe einen eigenen Namen verdient. Zu den Grundwörtern -macher, -haber, -halter, -gänger, -steller sind folgende Beispiele zu nennen:

$$
\begin{aligned}
& \text { Filmemacher, Liedermacher, Meinungsmacher } \\
& \text { Gewalthaber, Machthaber, Rechthaber, Liebhaber } \\
& \text { Fahrzeughalter, Tierhalter } \\
& \text { Fußgänger, Grenzgänger, Spaziergänger } \\
& \text { Schriftsteller, Eilzusteller, Gesuchssteller, Schausteller }
\end{aligned}
$$

Analogisch sind auch die Wörter Obstpflücker, Baumwollpflücker und Personenschützer entstanden.

Einige Berufsbezeichnungen enthalten Buchstabenwörter, die auf die Initialen, die Anfangsbuchstaben ihrer Vollformen, gekürzt worden sind. Buchstabenwörter mit zwei oder drei Initialen sind am häufigsten.

\begin{tabular}{|l|l|}
\hline Initialen-Komposita & Auflösung der Initialen \\
\hline IT -Lehrer, IT-Techniker, IT-Spezialist & Informationstechnik \\
\hline DB-Experte & Deutsche Bahn \\
\hline BND-Chef & Bundesnachrichtendienst \\
\hline CSU-Chef & Christlich-Soziale Union \\
\hline EU-Kommissar & Europäische Union \\
\hline Kfz-Mechaniker & Kraftfahrzeug \\
\hline PR-Profi & Public Relations \\
\hline SPD-Mann & Sozialdemokratische Partei Deutschlands \\
\hline TV-Moderatorin, TV-Techniker & Tele-Vision \\
\hline VW-Arbeiter & Volkswagen \\
\hline
\end{tabular}

Ein BWLer (Betriebswirtschaftler) entstand als eine einfache Ableitung durch das Suffix -er. Zwei ganz neue Wörter sind in der Studie "Deutsche Wortbildung" von Elke Donalies (DONALIES: 2007) zu lesen. Wenn sie die Sprachbenutzer bezeichnen will, gebraucht sie in ihren Erklärungen statt zwei selbständiger Wörter Hörer und Leser das Wort Hörerleser (S. 101): „Kurzwörter, die durch partielle Kürzung entstehen, beanspruchen den Hörerleser mehr als andere Wörter." Statt Sprecher und Schreiber spricht sie vom deutschen Sprecherschreiber (S. 95): „Außer den kombinierenden und den intern verändernden Wortbildungsarten nutzen deutsche Sprecherschreiber auch reduzierende Wortbildungsarten." 
Ein bekanntes Sprichwort sagt: Kleider machen Leute. Heutzutage scheint etwas anderes zu gelten: Titel machen Leute. Wer mit großen Firmen zu tun hat, insbesondere denjenigen mit anglophilen Titeln, der hat es oft schwer, die komplizierte Berufsbezeichnung zu verstehen. Warum werden die einfachsten Tätigkeiten mit aufgeblasenen Titeln versehen? Manche Firmen glauben, sie können einen eher weniger prestigeträchtigen Job für Bewerber anziehender machen, indem sie ihn mit blumigen Worten präsentieren. Nehmen wir als Beispiel ein paar konkrete „TopJobs mit Zukunft" aus der deutschen Presse:

Allround-Journalist - Berichterstattung für Printmedien, Fernsehen und Rundfunk. Analyst - bewertet Unternehmen und Märkte und erstellt Prognosen für deren Entwicklung.

Biotechnologe - entwickelt neue Produkte für die Pharma-Industrie aus Biomasse. CAD-Spezialist - entwirft Modelle von Möbeln, Karosserien oder Kleidung mit Hilfe von Computerprogrammen.

Controller - Planung und Kontrolle von Budgets großer Unternehmer.

Disponent - vermittelt und koordiniert Zeitarbeitskräfte.

Datenmanager - versucht, Ordnung in die unternehmerische Datenflut zu bringen. DV-Betreuer - sorgt für Instandhaltung betriebsinterner Computernetzwerke.

Entsorger - koordiniert Müllentsorgung und sucht neue Beseitigungswege.

Erlebnisgastronom - „Kochkünstler“sorgt dafür, dass Ausgehen Ereignischarakter bekommt.

Gebäudemanager - kümmert sich um Instandhaltung und Wartung großer Gebäude.

Haustechniker - Einbau und Wartung von High-Tech-Geräten in Gebäuden.

Hotliner - telefonische Beratung, Verkauf und technische Unterstützung.

IT-Lehrer-bildet Computerprofis aus.

Logistiker - steuert den gesamten Materialfluss im Unternehmen.

Mechatroniker - Montage und Wartung hochkomplexer Maschinen (MechanikerElektroniker)

Mediator - vermittelt in einem Ehestreit und sucht einen Ausweg aus der Ehekrise. Mediendidaktiker - entwickelt Software für multimediales Lernen mit dem PC.

Netzprojekteur - verwaltet, strukturiert und plant den Ausbau von Mobilfunknetzen.

Netzwerker - Aufbau von lokalen Computernetzwerken und Datenübertragung.

Online-Redakteur - gestaltet und verfasst die Inhalte von Internetseiten.

PR-Experte - sorgt für den perfekten öffentlichen Auftritt eines Unternehmens. R/3-Spezialist - Verkauf und Installation der weltweit gesuchten SAPDatenverarbeitungsprogramme.

Redenschreiber - verfasst die Reden von Politikern, Künstlern und Stars.

Screendesigner - gestaltet die Benutzeroberflächen von Computerprogrammen. 
Teletutor - persönlicher Lehrer, der sein Wissen via PC weitergibt. WEB-Konzeptionist - entwirft den Internetauftritt von Unternehmen. Wissensmanager - organisiert und bündelt das gesammelte Wissen eines Betriebes.

Die angeführten Beispiele von Berufsbezeichnungen wurden anhand von Zeitungsund Zeitschriftenlektüre gesammelt und analysiert. Die Wortbildungstypen sind gekennzeichnet durch eine typische Kombination von Wortbildungsmitteln sowie durch eine bestimmte Strukturbedeutung. Die beiden zentralen Wortbildungsarten des Deutschen sind kombinierende Verfahren, nämlich Komposition und explizite Derivation. Es ist zu erwarten, dass in Zusammenhang mit den Veränderungen in Gesellschaft, Wissenschaft und Wirtschaft immer neue Berufsbezeichnungen entstehen werden, die diverse Tätigkeiten auf dem heutigen Arbeitsmarkt benennen werden.

\section{Literatur:}

BRAUN (1997): Braun, Peter. Personenbezeichnungen. Tübingen: Max Niemeyer Verlag, 1997.

DONALIES (2007): Donalies, Elke. Basiswissen Deutsche Wortbildung. Tübingen, Basel: A. Francke Verlag, 2007.

FLEISCHER/BARZ (1995): Fleischer, Wolfgang/Barz, Irmhild. Wortbildung der deutschen Gegenwartssprache. Tübingen: Max Niemeyer Verlag, 2. Aufl., 1995.

GREWENDORF (1989): Grewendorf, Günther. Ergativity in German. Dordrecht, Providence: Foris Publications, 1989.

KARLÍK (2004): Karlík, Petr. Mikrosyntax českých deverbálních jmen, in: Sborník prací filozofické fakulty brnènské univerzity, A 52, 2004, 71-81.

KÜHN (1994): Kühn, Ingrid. Lexikologie. Tübingen: Max Niemeyer Verlag, 1994.

CLÉMENT (2000): Clément, Daniele. Linguistisches Grundwissen. Wiesbaden: Westdeutscher Verlag, 2. Aufl., 2000.

ALTMANN/KEMMERLING (2000): Altmann, Hans/Kemmerling, Silke. Wortbildung fürs Examen: Studien- und Arbeitsbuch. Wiesbaden: Westdeutscher Verlag, 2000.

\section{Quellen der angeführten Beispiel:}

www.focus.de

www.sueddeutsche.de

www.news.at 